\title{
Inhibition of Pim1 kinase reduces viral replication in primary bronchial epithelial cells
}

To the Editor:

Respiratory viral infections are responsible for $85 \%$ of asthma exacerbations, which in turn lead to increased morbidity and mortality, causing a high societal and economic burden $[1,2]$. Asthma patients have been found to be more susceptible to viral infections compared with non-asthmatic individuals [3]. It has been postulated that a reduced induction of apoptosis in virally infected airway epithelial cells from asthmatic patients might play a role in this increased susceptibility to viral infections [4]. Building on the role of apoptosis in respiratory viral infections, we tested whether the inhibition of a well-known survival pathway active in airway epithelial cells could reduce viral replication. To this end, we used an in vitro approach and infected primary bronchial epithelial cells (PBECs) from healthy volunteers with human rhinovirus (HRV)-16 in the absence or presence of a highly specific pharmacological inhibitor for Pim1 kinase [5]. Piml kinase is a constitutively active serine/threonine kinase known to be involved in cell survival by increasing the threshold for apoptosis $[6,7]$. We have shown previously that Pim1 kinase is highly expressed in the bronchial airway epithelium and that pharmacological inhibition of Pim1 kinase increases the sensitivity of bronchial epithelial cells to cell death upon challenge with cigarette smoke extract [8]. Therefore, we hypothesised that inhibition of Pim1 kinase activity in virally infected PBECs would enhance the onset of cell death, resulting in reduced viral replication.

Infection of monolayer cultures of PBECs with HRV-16 at a multiplicity of infection of 1 resulted in highly increased viral copy numbers $8 \mathrm{~h}$ and $24 \mathrm{~h}$ after infection compared with mock-infected controls (fig. 1a and b, respectively). Except for some low background signal, no viral RNA was detectable in either sham or ultraviolet light irradiated HRV-16 infected control PBECs or in PBECs exposed to the inhibitor in the absence of HRV-16. Interestingly, inhibition of Pim1 kinase activity significantly reduced viral RNA copy numbers, leading to a reduction in viral RNA load of $\sim 60-65 \%$ at both time points. The reduced viral replication upon inhibition of Pim1 kinase activity was further supported by a marked suppression of the amount of viral particles released by the PBECs $24 \mathrm{~h}$ after viral infection (fig. 1c). Viral particles were only observed in PBEC cultures infected with HRV-16. Inhibition of Pim1 kinase activity resulted in an almost 10-fold reduction in the amount of viral particles released from the PBECs. A comparable effect was observed with the ATP-binding-mimicking Pim1 kinase inhibitor SMI-4a, suggesting that the reduction in viral replication is Pim1 kinase specific (data not shown) [9]. Next, we aimed to test whether the observed reduced viral replication upon inhibition of Pim1 kinase activity was a consequence of an increased anti-viral response or enhanced induction of apoptosis. To this end, we first examined the anti-viral response and assessed the mRNA expression levels of the innate anti-viral cytokines interferon (IFN)- $\beta$ and interleukin (IL)-29, $8 \mathrm{~h}$ after infection. Although expression levels were relatively low in the PBEC monolayer cultures, in agreement with KHAITOv et al. [10], inhibition of Pim1 kinase activity resulted in increased mRNA expression of IFN- $\beta$ (fig. 1d) and IL-29 (fig. 1e) compared to the non-treated HRV-16 infected control cultures. Evaluating the activation of the anti-viral inflammatory response by analysing the mRNA expression of the IFN-inducible genes IFN $\gamma$-induced protein (IP)-10 and RANTES, we observed a significantly decreased expression of both IP-10 (fig. 1f) and RANTES (fig. 1g) in HRV-16 infected PBEC cultures treated with the Pim1 kinase inhibitor compared to control cultures. As shown in figure $1 \mathrm{~h}$ and $\mathrm{i}, \mathrm{IP}-10$ and RANTES protein levels in supernatant were extremely low and we were not able to detect IP-10 or RANTES in all samples, which might be a consequence of the relatively short half-life, as determined for RANTES [11]. While we could not perform statistical evaluation of these data, IP-10 (fig. 1h) and RANTES (fig. 1i) protein levels seem to follow mRNA expression levels, with increased protein levels upon infection with HRV-16 and a subsequent decrease in protein levels upon concurrent inhibition of Pim1 kinase activity. Since the increased innate anti-viral response (mRNA expression of type I and III IFNs) observed upon inhibition of Pim1 kinase in virally infected PBECs did not correspond with an increased activation of the anti-viral inflammatory response, we next examined the role of cell death in reduced viral replication upon inhibition of Pim1 kinase activity. Based on the reduced viral RNA 


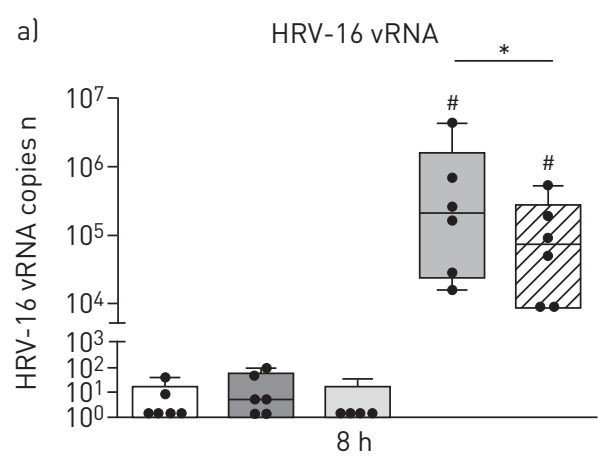

b)

HRV-16 vRNA

c)

HRV-16 viral load
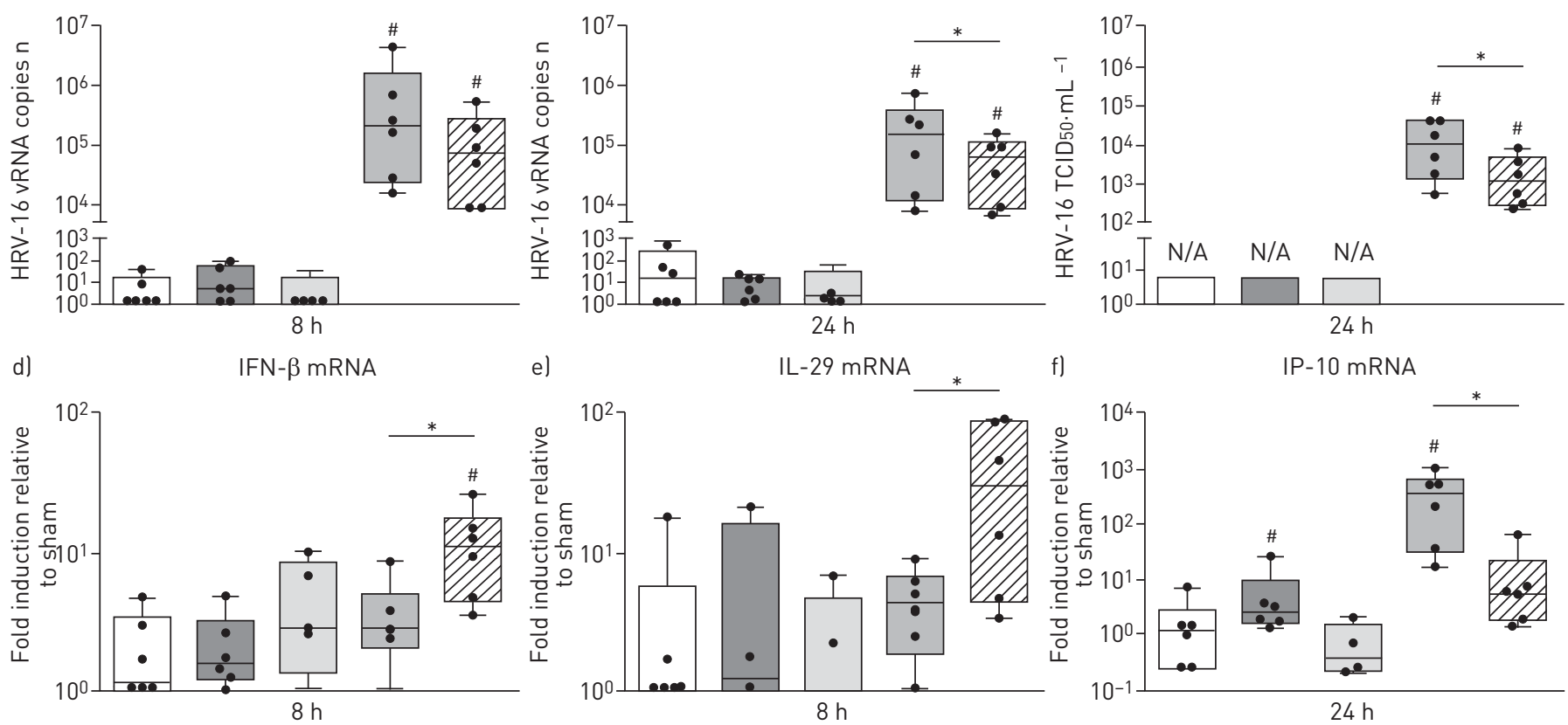

e)

IL-29 mRNA

f)

IP-10 mRNA
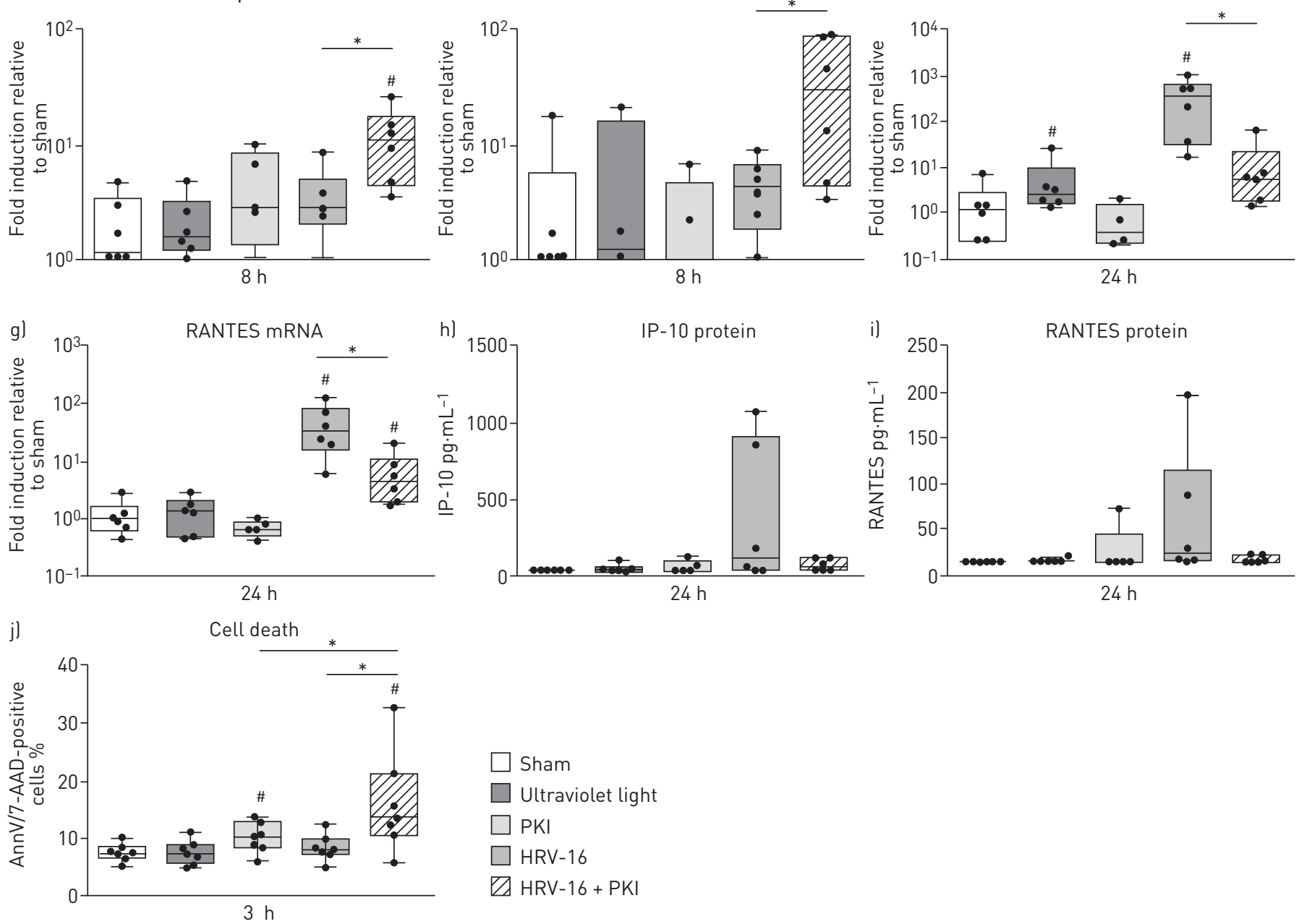

FIGURE 1 Infection of monolayer cultures of primary bronchial epithelial cells with human rhinovirus (HRV)-16 in the presence of a specific pharmacological Pim1 kinase inhibitor (PKI) reduces viral replication by augmenting the onset of cell death. Viral (v)RNA copy numbers were determined using reverse transcriptase (RT)-quantitative (q)PCR a) $8 \mathrm{~h}$ and b) $24 \mathrm{~h}$ after infection. c) Release of viral particles was measured by $50 \%$ tissue culture infective dose (TCID50) $24 \mathrm{~h}$ after infection. mRNA expression of the innate anti-viral genes d) interferon (IFN)- $\beta$ and e) interleukin (IL)-29 $8 \mathrm{~h}$ after infection was assessed using RT-qPCR. mRNA expression of the IFN-inducible genes f) IFN $\gamma$-induced protein (IP)-10 and g) RANTES $24 \mathrm{~h}$ after infection was measured using RT-qPCR. Protein levels of h) IP-10 and i) RANTES $24 \mathrm{~h}$ after infection were analysed using commercially available ELISA (R\&D Systems, Abingdon, UK). j) The amount of cell death $3 \mathrm{~h}$ after infection was assessed by analysing annexin V (AnnV) and 7-amino-actinomycin D (AAD)-positive cells using flowcytometry. The experiments were repeated six or seven times. Data are presented as median and range. Statistical significance was tested using the paired two-sided Wilcoxon matched pairs signed rank test. N/A: not applicable. : $\mathrm{p}<0.05 ;{ }^{*}: \mathrm{p}<0.05$ compared to sham-infected controls.

replication in the presence of the Pim1 kinase inhibitor observed at $8 \mathrm{~h}$ post-infection, we sought to analyse the induction of cell death at earlier time points. This was determined experimentally and shown to be optimal $3 \mathrm{~h}$ after infection. Therefore, we determined the induction of cell death upon infection with HRV-16 by analysing the presence of phosphatidylserine on the outer cell membrane and loss of cell membrane integrity $3 \mathrm{~h}$ after infection. As shown in figure $1 \mathrm{j}$, no significant differences could be observed 
in cell death when cells were infected with HRV-16 compared with controls. However, cells infected with HRV-16 in the presence of the Pim1 inhibitor showed a significant increase in the amount of cell death compared with cells infected with HRV-16 alone or treated with the Pim1 inhibitor in the absence of HRV-16 (fig. 1j), suggesting that Pim1 inhibition sensitises the PBECs to apoptosis induction upon HRV-16 infection.

This study shows for the first time that inhibition of Pim1 kinase activity reduces viral replication and release of viral particles in cultured PBECs from healthy volunteers by enhancing the induction of cell death upon viral infection. The observed role of cell death in viral infections is in line with previous studies showing that the early apoptotic response is an important feature of the anti-viral response, limiting viral replication and subsequent viral spread $[4,12]$. While decreased cell survival alone could explain the reduction in viral replication, inhibition of Pim1 kinase also resulted in a slight increase in mRNA expression of IFN- $\beta$ and IL-29, $8 \mathrm{~h}$ after infection with HRV-16. However, mRNA expression levels of IFN- $\beta$ and IL-29 in virally infected PBEC cultures treated with the Pim1 kinase inhibitor are very low and were not sufficient to enhance levels of the IFN-inducible genes IP-10 and RANTES, $24 \mathrm{~h}$ after infection. The levels of IP-10 and RANTES probably follow the amount of virus present in the cells, which is markedly reduced upon inhibition of Piml kinase activity (fig. 1b, c) [13]. Consequently, we feel that the inhibition of viral replication by inhibition of Pim1 kinase is largely achieved through its effect on reduced cell survival of HRV-16 infected cells. Taken together, our findings imply that inhibition of Pim1 kinase inhibits viral replication and viral particle release from HRV-infected PBECs. Therefore, Pim1 inhibition could be a new therapeutic approach in the treatment of viral-induced asthma exacerbations. Further work is now required to assess the effect of Pim1 kinase inhibition in PBECs from patients with severe asthma and to explore the underlying mechanisms of suppression of viral replication and the role of an enhanced innate anti-viral response upon inhibition of Pim1 kinase activity. Also of interest is testing the broader potential of inhibition of Pim1 kinase activity to affect the replication of other respiratory viruses, such as influenza and respiratory syncytial virus, also commonly detected in virally induced asthma exacerbations [1].

0 @ERSpublications

Inhibition of Pim1 kinase activity reduces viral replication by inducing the onset of bronchial epithelial cell death http://ow.ly/IJWGD

Maaike de Vries ${ }^{1,2,3}$, Natalie P. Smithers ${ }^{2}$, Peter H. Howarth ${ }^{4}$, Martijn C. Nawijn ${ }^{1,3}$ and Donna E. Davies ${ }^{2,4}$

${ }^{1}$ Laboratory of Experimental Pulmonology and Inflammation Research, Dept of Pathology and Medical Biology, University of Groningen, University Medical Center Groningen, Groningen, The Netherlands. ${ }^{2}$ Brooke Laboratory, Academic Unit of Clinical and Experimental Sciences, University of Southampton, Faculty of Medicine, University Hospital Southampton, Southampton, UK. ${ }^{3}$ GRIAC Research Institute, University of Groningen, University Medical Center Groningen, Groningen, The Netherlands. ${ }^{4}$ National Institute for Health Research Respiratory Biomedical Research Unit, University Hospital Southampton, Southampton, UK.

Correspondence: M. de Vries, Laboratory of Experimental Pulmonology and Inflammation Research, Dept of Pathology and Medical Biology, University Medical Center Groningen, Hanzeplein 1, 9713 GZ, The Netherlands.

E-mail: m.de.vries04@umcg.nl

Received: Nov 062014 | Accepted after revision: Jan 272015 | First published online: March 052015

Conflict of interest: Disclosures can be found alongside the online version of this article at erj.ersjournals.com

Support statement: This work was supported by European Respiratory Society Fellowship LTRF 2013-2135 to Maaike de Vries, a shared University of Groningen and University of Southampton PhD studentship (GUIDE PhD studentship to Maaike de Vries) and Medical Research Council (UK) grant number G0900453. Funding information for this article has been deposited with FundRef.

Acknowledgements: The authors would like to thank Juerg Schwaller (University Children's Hospital of Basel, Basel, Switzerland) and Andrew Kraft (Medical University of South Carolina, Charleston, SC, USA) for providing the Pim1 inhibitors K00135 and SMI-4a, respectively, and Robert Ridley and Graham Berreen (University Hospital Southampton, Southampton, UK) for the technical assistance with the primary bronchial epithelial cell cultures.

\section{References}

1 Jackson DJ, Sykes A, Mallia P, et al. Asthma exacerbations: origin, effect, and prevention. J Allergy Clin Immunol 2011; 128: 1165-1174.

2 O'Byrne PM. Therapeutic strategies to reduce asthma exacerbations. J Allergy Clin Immunol 2011; 128: 257-263.

3 Cakebread JA, Xu Y, Grainge C, et al. Exogenous IFN- $\beta$ has antiviral and anti-inflammatory properties in primary bronchial epithelial cells from asthmatic subjects exposed to rhinovirus. J Allergy Clin Immunol 2011; 127: 1148-1154.

4 Wark PA, Johnston SL, Bucchieri F, et al. Asthmatic bronchial epithelial cells have a deficient innate immune response to infection with rhinovirus. J Exp Med 2005; 201: 937-947. 
5 Pogacic V, Bullock AN, Fedorov O, et al. Structural analysis identifies imidazo[1,2-b]pyridazines as PIM kinase inhibitors with in vitro antileukemic activity. Cancer Res 2007; 67: 6916-6924.

6 Macdonald A, Campbell DG, Toth R, et al. Pim kinases phosphorylate multiple sites on Bad and promote 14-3-3 binding and dissociation from Bcl-XL. BMC Cell Biol 2006; 7: 1.

7 Nawijn MC, Alendar A, Berns A. For better or for worse: the role of Pim oncogenes in tumorigenesis. Nat Rev Cancer 2011; 11: 23-34.

8 de Vries M, Heijink IH, Gras R, et al. Pim1 kinase protects airway epithelial cells from cigarette smoke-induced damage and airway inflammation. Am J Physiol Lung Cell Mol Physiol 2014; 307: L240-L251.

9 Lin YW, Beharry ZM, Hill EG, et al. A small molecule inhibitor of Pim protein kinases blocks the growth of precursor T-cell lymphoblastic leukemia/lymphoma. Blood 2010; 115: 824-833.

10 Khaitov MR, Laza-Stanca V, Edwards MR, et al. Respiratory virus induction of alpha-, beta- and lambda-interferons in bronchial epithelial cells and peripheral blood mononuclear cells. Allergy 2009; 64: 375-386.

11 Hillyer P, Male D. Expression of chemokines on the surface of different human endothelia. Immunol Cell Biol 2005; 83: 375-382.

12 Takaoka A, Hayakawa S, Yanai H, et al. Integration of interferon-alpha/beta signalling to p53 responses in tumour suppression and antiviral defence. Nature 2003; 424: 516-523.

13 Spurrell JC, Wiehler S, Zaheer RS, et al. Human airway epithelial cells produce IP-10 (CXCL10) in vitro and in vivo upon rhinovirus infection. Am J Physiol Lung Cell Mol Physiol 2005; 289: L85-L95.

\section{Pathogenicity of individual rhinovirus species during exacerbations of cystic fibrosis}

To the Editor:

Pulmonary exacerbations in patients with cystic fibrosis (CF) accelerate deterioration of lung function and cause increased morbidity and mortality [1]. Viruses have been identified in up to $60 \%$ of CF related pulmonary exacerbations with a higher prevalence in children [2-5]. FLIGHT et al. [4] identified viruses in $40 \%$ of exacerbations in adult patients with CF; viral PCR was performed on nasal, throat, and sputum specimens. We routinely undertake viral throat swabs in all adult patients receiving intravenous antibiotics as part of prospective clinical surveillance, with data automatically uploaded to the electronic patient record (EPR). Patients with self-limiting exacerbations are not screened. In our selected cohort, viral PCR was positive in $9.2 \%$ of cases and a positive result is associated with longer duration of treatment, increased inflammatory response, and less recovery of lung volumes [3].

In both studies human rhinovirus (HRV) accounted for $>70 \%$ of the viruses identified $[3,4]$.

HRVs are common respiratory pathogens that traditionally, it has been felt, result in self-limiting and mild upper respiratory symptoms. HRVs are members of the Enterovirus genus from the family Picornaviridae. With the advent of molecular methods of detecting HRV a great diversity of HRVs has been identified [6]. This includes HRV species $\mathrm{C}$, which is unculturable and failed to be identified by previous viral isolation methods. Three species of HRV are now known, these are HRV A, HRV B, and HRV C. Within each species are many types, which are classified according to sequence divergence in the VP1 region that codes for a capsid protein [6].

Recent studies have implicated certain species of HRV as being more pathogenic. In childhood asthma, exacerbations due to HRV-C have been associated with more severe exacerbations than other HRV groups $[7,8]$. In stable children with CF the presence of HRV-C or HRV-A2 is associated with an increased frequency of exacerbations [9]. Studies in adult patients with CF have investigated the role of individual HRV species, although, they have not been specifically designed to assess the effect on clinical outcomes following exacerbations. FLIGHT et al [4] performed typing on HRV positive samples from 42 clinic visits involving 27 patients; HRV-A was most frequently identified and appeared to induce more upper respiratory symptoms than HRV-B. The group identified HRV-C in only $4.8 \%$ of patients. In contrast, GOFFARD et al. [5] detected HRV-C in 44\% of pulmonary exacerbations, although the numbers were small. 